The Astrophysical Journal, 631:L117-L120, 2005 October 1

(C) 2005. The American Astronomical Society. All rights reserved. Printed in U.S.A.

\title{
BINARY ENCOUNTERS WITH SUPERMASSIVE BLACK HOLES: ZERO-ECCENTRICITY LISA EVENTS
}

\author{
M. Coleman Miller, ${ }^{1}$ Marc Freitag, ${ }^{2}$ Douglas P. Hamilton, ${ }^{1}$ and Vanessa M. Lauburg \\ Received 2005 July 6; accepted 2005 August 22; published 2005 September 15
}

\begin{abstract}
Current simulations of the rate at which stellar-mass compact objects merge with supermassive black holes (called extreme mass ratio in-spirals, or EMRIs) focus on two-body capture by emission of gravitational radiation. The gravitational wave signal of such events will likely involve a significant eccentricity in the sensitivity range of the Laser Interferometer Space Antenna (LISA). We show that tidal separation of stellar-mass compact object binaries by supermassive black holes will instead produce events whose eccentricity is nearly zero in the LISA band. Compared to two-body capture events, tidal separations have a high cross section and result in orbits that have a large pericenter and small apocenter. Therefore, the rate of interactions per binary is high, and the resulting systems are very unlikely to be perturbed by other stars into nearly radial plunges. Depending on the fraction of compact objects that are in binaries within a few parsecs of the center, the rate of low-eccentricity LISA events could be comparable to or larger than the rate of high-eccentricity events.
\end{abstract}

Subject headings: binaries: general — galaxies: nuclei — gravitational waves — relativity

\section{INTRODUCTION}

Extreme mass ratio in-spirals (EMRIs) of stellar-mass compact objects into supermassive black holes are key targets for the Laser Interferometer Space Antenna (LISA). From the fundamental physics standpoint, these events are expected to provide the best available mapping of the spacetime around a rotating black hole (Ryan 1995, 1997; Hughes 2003). Astrophysically, they may well reveal the numbers of supermassive black holes in a mass range $\left(\sim 10^{5}-10^{7} M_{\odot}\right)$ that is difficult to probe otherwise (e.g., Greene \& Ho 2004).

Current studies of EMRI rates and properties (Hils \& Bender 1995; Sigurdsson \& Rees 1997; Miralda-Escudé \& Gould 2000; Freitag 2001, 2003; Ivanov 2002; Hopman \& Alexander 2005) have focused exclusively on the capture of compact objects by the emission of gravitational radiation during a close pass. That is, a compact object (for example, a $10 M_{\odot}$ black hole) passes close to the central supermassive black hole (SMBH) and emits gravitational waves that shrink its orbit significantly. The black hole then continues to orbit, and if its motion is not perturbed significantly by interactions with other stars, then it eventually spirals into the SMBH. When the orbit becomes detectable with LISA, it has a significant eccentricity of typically $e \sim 0.5-0.9$ (Freitag 2003; Hopman \& Alexander 2005; but see Ivanov 2002).

Here we consider a different process, in which a stellar-mass binary containing a compact object comes close enough to the SMBH that the binary is tidally separated, leaving one object bound to the SMBH and the other almost always ejected to infinity at high speed. Tidal separation was discussed recently by Pfahl (2005) as a way to fuel intermediate-mass black holes and has been considered as a method to produce high-velocity stars (Hills 1988, 1991; Yu \& Tremaine 2003; Brown et al. 2005) and as a possible way to deposit high-mass stars close to Sgr A* (Gould \& Quillen 2003). It was also listed by Hils \& Bender (1995) and Freitag \& Benz (2002) as a mechanism

\footnotetext{
${ }^{1}$ Department of Astronomy, University of Maryland at College Park, College Park, MD 20742-2421; miller@astro.umd.edu, hamilton@astro.umd.edu, vanessa@astro.umd.edu.

${ }^{2}$ Department of Physics and Astronomy, Northwestern University, 2131 Sheridan Road, Evanston, IL 60208-2900; m-freitag@northwestern.edu.
}

to be examined in the EMRI context, but to our knowledge it has not yet been explored quantitatively.

The key point about this process is that, unlike in the twobody capture scenario, no energy needs to be dissipated in order to have a capture. As a result, capture can occur at much larger radii than is possible in the two-body case: for example, a binary with a semimajor axis of tenths of an AU can be captured at pericenter distances of tens of $\mathrm{AU}$ relative to the $\mathrm{SMBH}$, compared with the $\sim 0.1$ AU that is required for two-body capture. In addition, the semimajor axis of the resulting bound object will be modest, perhaps tens of times the pericenter distance (Hills 1991; Pfahl 2005). EMRIs formed in this way are therefore relatively immune to perturbations of their orbits that could cause them to plunge directly into the SMBH (which lowers rates significantly for EMRIs formed by two-body capture; see Hils \& Bender 1995 and Hopman \& Alexander 2005). Combined with the higher cross section, this suggests that the overall rate of EMRIs could have an important contribution from the tidal separation of binaries, even if only a few percent of compact objects are in binaries. In addition, the high pericenter after capture implies that when tidal separation EMRIs are detectable with LISA, they will have eccentricities close to zero (there might also be independent paths to low eccentricity, such as the production of black holes in an accretion disk and their subsequent advection to the SMBH; see Levin 2003).

In $\S 2$ we discuss this process in more quantitative detail. In $\S 3$ we list some of the questions that will have to be answered to get more specific predictions of relative rates, and to interpret LISA observations when they arrive.

\section{TIDAL SEPARATION AND EMRIs}

\subsection{Capture Processes}

To evaluate the tidal separation scenario, let us first recall the process of two-body capture. Suppose that a point mass $m$ orbits an SMBH of mass $M \gg m$ with an orbital speed $v_{\infty}$ at apocenter (assumed to be at a large distance). Its orbit will be modified significantly if, during its motion, it releases $\gtrsim \frac{1}{2} m v_{\infty}^{2}$ of energy 
in gravitational radiation. From Quinlan \& Shapiro (1989), this condition implies a pericenter distance

$$
\begin{aligned}
r_{p}<r_{p, \mathrm{GW}} \approx & 0.13 \mathrm{AU}\left(m / 10 M_{\odot}\right)^{2 / 7}\left(M / 10^{6} M_{\odot}\right)^{5 / 7} \\
& \times\left(v_{\infty} / 60 \mathrm{~km} \mathrm{~s}^{-1}\right)^{-4 / 7} .
\end{aligned}
$$

We have scaled by $60 \mathrm{~km} \mathrm{~s}^{-1}$ because this is roughly the velocity dispersion inferred for a galaxy with a central black hole mass of $10^{6} M_{\odot}$ (Merritt \& Ferrarese 2001; Tremaine et al. 2002; Barth et al. 2005). The time required to spiral into the SMBH would then be much less than a Hubble time, except that other stars perturb the orbit significantly (see $\S 2.2$ ). The gravitational radius is $r_{g} \equiv G M / c^{2} \approx 0.01 \mathrm{AU}\left(M / 10^{6} M_{\odot}\right)$. Therefore,

$$
\begin{aligned}
r_{p, \mathrm{GW}} / r_{g} \approx & 13\left(m / 10 M_{\odot}\right)^{2 / 7}\left(M / 10^{6} M_{\odot}\right)^{-2 / 7} \\
& \times\left(v_{\infty} / 60 \mathrm{~km} \mathrm{~s}^{-1}\right)^{-4 / 7} .
\end{aligned}
$$

For comparison, the radius of the innermost stable circular orbit around a nonrotating SMBH is $6 r_{g}$. As another comparison, detection of an EMRI with LISA will be very difficult if the gravitational wave frequency is less than $f_{\mathrm{GW}} \sim 2-3 \mathrm{mHz}$, because at lower frequencies there is strong unresolvable foreground noise due to double white dwarf binaries in our Galaxy (Bender \& Hils 1997; Nelemans et al. 2001; Farmer \& Phinney 2003). For a circular orbit, the gravitational wave frequency is double the orbital frequency (Peters \& Mathews 1963). At $2 \mathrm{mHz}$, then, the radius of a circular orbit is $r(2 \mathrm{mHz}) \approx$ $10 r_{g}\left(M / 10^{6} M_{\odot}\right)^{-2 / 3}$. Therefore, a stellar-mass compact object needs to go very deep into the potential well of an SMBH to be captured or to be observed with LISA. As a consequence, although the orbit circularizes due to emission of gravitational radiation (Peters 1964), the eccentricity in the LISA band is still $e \sim 0.5-0.9$.

Now consider tidal separation. Suppose that a binary with a total mass $m$ and semimajor axis $a$ moves toward a supermassive black hole of mass $M$. If the orbit has a pericenter distance less than

$$
\begin{aligned}
r_{\text {tide }} & \approx(3 M / m)^{1 / 3} a \\
& \approx 7 \mathrm{AU}\left(M / 10^{6} M_{\odot}\right)^{1 / 3}\left(m / 10 M_{\odot}\right)^{-1 / 3}(a / 0.1 \mathrm{AU}),
\end{aligned}
$$

then the binary will be separated by the tidal field of the SMBH. Note that the numerical factor in the cube root is correct for a prograde binary on a circular orbit around the SMBH; it changes to 4 for weakly hyperbolic prograde orbits and roughly half this for retrograde orbits (Hamilton \& Burns 1991, 1992). We scale $a$ by 0.1 AU because such a binary is tight enough to survive three-body encounters but wide enough to avoid rapid merger by gravitational radiation (see $\S 3$ for further discussion).

For an initially hard circular binary with component masses $10 M_{\odot}$ and $10 M_{\odot}$ in a hyperbolic pass by a $10^{6} M_{\odot} \mathrm{SMBH}$, our numerical simulations suggest that the typical eccentricity is $e \sim 0.98$ after capture, consistent with the results of Hills (1991) and Pfahl (2005), who focused on tidal separation of main-sequence binaries. For an initial binary separation of $a=0.1 \mathrm{AU}$, the typical pericenter distance after capture is a few AU, and the typical apocenter distance is a few hundred $\mathrm{AU}$; both are proportional to the semimajor axis of the original binary. We also simulated the tidal separation of initially hard circular binaries with component masses $10 M_{\odot}$ and $1 M_{\odot}$ around a $10^{6} M_{\odot} \mathrm{SMBH}$, representing, for example, a binary with a black hole and a white dwarf or a black hole and a neutron star. We find that only a small fraction of encounters lead to ejection of both objects or survival of the binary, the rest resulting in capture of one object and ejection of the other. In $\sim 40 \%$ of the captures, the $10 M_{\odot}$ object becomes bound to the SMBH, with an apocenter distance that is a factor of a few larger than for the $10 M_{\odot}$ and $10 M_{\odot}$ simulations (as is expected given the smaller energy transfer from the $1 M_{\odot}$ object; see Pfahl 2005 for an analytic discussion). In the remaining $\sim 60 \%$ of the captures, the $1 M_{\odot}$ compact object is captured, which also leads to an extreme mass ratio in-spiral but a weaker one than the $10 M_{\odot}$ and $10^{6} M_{\odot}$ coalescence would produce.

Because the pericenter distance from binary capture is $\gg r_{g}$, the orbit circularizes dramatically by emission of gravitational radiation and typically has $e<0.01$ in the LISA sensitivity band, in sharp contrast to EMRIs produced by capture of singles.

\subsection{Effects of Nuclear Stellar Dynamics}

The motion of a binary must be close to radial to be captured. For example, a binary with semimajor axis $a \sim 1$ AU could be captured if it passed within $\sim 100 \mathrm{AU}$ of the SMBH, but this is tiny compared to the distance of a few parsecs from the SMBH where most binaries presumably lie. It is therefore important to map out some of the dynamical processes that will affect the injection into these orbits. These are discussed in detail by many authors (e.g., Frank \& Rees 1976; Lightman \& Shapiro 1977; Magorrian \& Tremaine 1999; Syer \& Ulmer 1999), so here we simply quote the results.

A supermassive black hole of mass $M$ will dominate the dynamics out to the "radius of influence"

$$
r_{\text {infl }}=G M / \sigma_{0}^{2} \approx 1 \operatorname{pc}\left(M / 10^{6} M_{\odot}\right)\left(60 \mathrm{~km} \mathrm{~s}^{-1} / \sigma_{0}\right)^{2},
$$

where $\sigma_{0}$ is the velocity dispersion of stars far outside this radius. At radii $r>r_{\text {inf }}$, a constant velocity dispersion implies a stellar-mass density $\rho \sim r^{-2}$, whereas at $r<r_{\text {infl }}$ the density can take a different slope $\rho \sim r^{-\gamma}$, for example, $\gamma=3 / 2$ or $\gamma=7 / 4$ (e.g., Bahcall \& Wolf 1976; Young 1980).

For $r<r_{\text {infl }}$, the orbital time is $t_{\text {orb }}=2 \pi\left(r^{3} / G M\right)^{1 / 2}$, whereas for $r>r_{\text {inf }}, t_{\text {orb }}=2 \pi\left(r / r_{\text {infl }}\right)\left(G M / \sigma_{0}^{3}\right)$. The relaxation time is the time required for the velocity of a star to change by of order itself (in magnitude or direction), by deflections due to twobody encounters. The local relaxation time for a compact object of mass $m_{\mathrm{CO}}$ interacting with stars of average mass $\langle m\rangle$ is (Spitzer 1987)

$$
t_{\mathrm{rlx}}(r)=\frac{0.339}{\ln \Lambda} \frac{\sigma^{3}(r)}{G^{2}\langle m\rangle m_{\mathrm{CO}} n(r)} .
$$

Here $\sigma(r)$ is the local velocity dispersion (equal to the orbital speed when $\left.r<r_{\text {infl }}\right), n(r)$ is the local number density, and $\ln \Lambda \sim 10$ is the Coulomb logarithm. Inside $r_{\text {infl }}$, the relaxation time is roughly constant.

For a bound object on a very eccentric orbit, $e \approx 1$, the angular momentum is much less than the angular momentum of a circular orbit with the same semimajor axis. Therefore, the angular momentum only needs to change slightly to make an order unity difference in the orbit. This timescale is $t_{J}(r, e) \approx(1-e) t_{\mathrm{rlx}}(r)$ (e.g., Hopman \& Alexander 2005).

For a given position $R$ and speed $V$, the loss cone is defined as the set of directions of the velocity $\boldsymbol{V}$ leading to such small 
pericenter distances that the object of interest is removed from the system. In the full loss cone regime, for which $t_{J}<t_{\text {orb }}$ (where $t_{J}$ is evaluated for the angular momentum corresponding to the loss cone), objects that enter the loss cone and are removed are immediately replaced, within an orbital time, by objects that are deflected in from other orbits. In this regime, an object that starts down the loss cone is likely to be deflected out of the cone during the orbit. In the empty loss cone regime, for which $t_{J}>t_{\mathrm{orb}}$, replacement of objects through the loss cone has to occur over a relaxation time. If the number of objects per radius (assuming spherical symmetry) is $d N / d r$ and the angle subtended by the loss cone at radius $r$ is $\theta_{\mathrm{LC}}(r)$, then the approximate capture rates in the full and empty loss cone regimes are (see Syer \& Ulmer 1999)

$$
\begin{gathered}
d \dot{N}_{\text {full }} / d r \sim \theta_{\mathrm{LC}}^{2}(r)(d N / d r) / t_{\text {orb }}, \\
d \dot{N}_{\mathrm{empty}} / d r \sim(d N / d r) /\left\{\ln \left[1 / \theta_{\mathrm{LC}}^{2}(r)\right] t_{\mathrm{rlx}}(r)\right\} .
\end{gathered}
$$

Far from the SMBH the loss cone is full, whereas close it is empty. For the binaries, the full/empty transition radius (which defines the "critical radius") is comparable to the radius of influence, whereas for the singles the transition occurs at $\sim 10 \%$ of the radius of influence. The merger rate is dominated by the region near the critical radius (see, e.g., Frank \& Rees 1976). The smaller critical radius for singles partially compensates for the much larger cross section of the binaries, and for $\gamma=3 / 2$ the net merger rate enhancement turns out to be roughly a factor of 10 in favor of the binaries.

As pointed out by Hils \& Bender (1995) and analyzed by Hopman \& Alexander (2005), there is an additional major effect. A single compact object captured by gravitational radiation emission typically has a very large apocenter distance, often on the order of tenths of a parsec or more. As a result, even after it has first been captured, it has a chance to be perturbed in the next orbit. Sometimes, a perturbation will cause the orbit to be so close to radial that the object plunges straight into the SMBH. Although this does not affect the merger rate, such objects do not contribute to the LISA event rate, because they plunge before their orbital period has become shorter than $\approx 10^{3}-10^{4}$ s. Hopman \& Alexander (2005) estimate that $\sim 80 \%-$ $90 \%$ of the potential EMRI events are lost in this fashion. This effect amounts to reducing significantly the volume from which LISA-detectable EMRIs can originate, which therefore decreases the observed rate. Note, however, that mass segregation of black holes into a dense subcluster may reduce the impact of this effect (E. S. Phinney 2005, private communication).

In contrast, in-spirals produced by separation of binaries are not susceptible to this effect. The reason is that, as discussed in $\S 2.1$, the apocenter distance is usually only tens of times the pericenter distance; hence, $t_{J} \gg t_{\text {orb }}$. As a result, we expect that any perturbations will be gradual; hence, a decrease in the pericenter distance will produce greater gravitational radiation emission and thus circularization rather than a plunge.

Processes that enhance angular momentum diffusion, such as resonant relaxation (Rauch \& Tremaine 1996) and interactions in triaxial potentials (Holley-Bockelmann et al. 2002; Poon \& Merritt 2002; Merritt \& Poon 2004), will tend to push the full loss cone regime to smaller radii, which will enhance rates moderately for both binaries and singles. However, assessment of the net effects will require detailed calculations (cf. Rauch \& Ingalls 1998, who show that the total rate of stellar tidal disruptions is at most doubled by resonant relaxation, because the rate bottleneck is elsewhere).

\section{DISCUSSION AND CONCLUSIONS}

In this Letter we have focused on interactions that leave an $\sim 10 M_{\odot}$ black hole in orbit around a $10^{6} M_{\odot}$ SMBH. We note that the same process will also enhance rates for neutron stars and white dwarfs around $10^{6} M_{\odot}$ black holes, likely by an even larger factor, because we see from equation (2) that direct capture of lower mass objects at a given speed requires an even closer passage to the SMBH. There may also be a moderate effect for intermediate-mass black holes (IMBHs) of $\sim 10^{3} M_{\odot}$ in dense stellar clusters (see Miller \& Colbert 2004 for an overview of the evidence for IMBHs and their association with clusters), as discussed by Pfahl (2005). This could lead to detection with LISA of BH-IMBH orbits (see Baumgardt et al. 2004 for discussion of gravitational radiation from direct capture onto IMBHs in clusters) or orbits of disrupted stars around IMBHs (Hopman \& Portegies Zwart 2005), although only the nearest sources are likely to be seen (Will 2004). In addition, the loss cone formalism is unlikely to be directly relevant here because for $M \lesssim 10^{3} M_{\odot}$, the wandering radius of the black hole is comparable to or larger than its radius of influence (see Merritt 2001), and hence the interactions need to be treated as independent binary-single encounters (see Gültekin et al. 2004 for a recent application to intermediate-mass black holes).

It is not trivial to estimate the absolute rate of EMRI captures by the mechanism we describe, because of the number of processes involved. For example, the fraction of black holes in binaries is especially important (see Muno et al. 2005 for a recent discussion in the context of the Galactic center). This fraction depends on (1) the fraction of binaries that survive stellar evolution (Belczynski et al. 2004), (2) the fraction of those binaries that survive interactions in the dense stellar environment of the galactic nucleus (Ivanova et al. 2005), and (3) the fraction of initially solitary black holes that acquire companions by threebody interactions before they are captured by the SMBH. An important output from the combination of these processes is (4) the distribution of semimajor axes of binaries containing black holes, because very tight binaries ( $a \lesssim 0.05$ AU for a 10 and $10 M_{\odot}$ binary) could merge by gravitational radiation before being separated by the SMBH, and black holes in very wide binaries (more than a few AU) could end up after separation with semimajor axes so large (more than $\sim 0.1 \mathrm{pc}$ ) that perturbations during a single dynamical time drop them into plunge orbits, making them undetectable with LISA (Hopman \& Alexander 2005). Mass segregation will tend to move black hole binaries to regions of higher density and higher velocity dispersion, where three-body interactions are important; hence, we must also compute (5) the evolution of the semimajor axis and companion mass as a function of time, versus the probability of capture by the SMBH as a function of time, to estimate the true distribution of semimajor axes after capture, and hence the subsequent evolution of the orbit under the influence of relaxation and gravitational radiation. All of these processes will require careful computation in future work.

Without knowing the absolute rate, we can parameterize the ratio of the rate of captures due to binary separation to the rate of captures due to singles as

$$
\frac{\dot{N}_{\text {binary }}}{\dot{N}_{\text {single }}}=\frac{f_{b} \mathcal{R}_{\text {binary }}\left\langle f_{\text {binary, LISA }}\right\rangle}{f_{s} \mathcal{R}_{\text {single }}\left\langle f_{\text {single, } \text { LISA }}\right\rangle},
$$


where $f_{b}$ is the fraction of compact objects that are in binaries that are neither too tight nor too wide (see above); $f_{s}$ is the fraction that are single; $\mathcal{R}_{\text {binary }}$ is the total rate of tidal separations per binary; $\mathcal{R}_{\text {single }}$ is the total rate of gravitational radiation captures per single; $\left\langle f_{\text {binary, LISA }}\right\rangle$ is the overall fraction of binary sources captured in orbits tight enough to spiral into the LISA band within a Hubble time; and $\left\langle f_{\text {single, LISA }}\right\rangle$ is the overall fraction of captured singles that end up detectable with LISA (rather than being perturbed into plunge orbits). Our current best guesses are $\mathcal{R}_{\text {binary }} / \mathcal{R}_{\text {single }} \sim 10$ and $\left\langle f_{\text {binary, LISA }}\right\rangle /\left\langle f_{\text {single, LISA }}\right\rangle \sim 1-10$. Therefore, if the steady state binary fraction (reduced by merging at small semimajor axes and by ionizations at large semimajor axes) is $f_{b}>0.01-0.1$, EMRIs from binaries could dominate the total rates.

We thank the Aspen Center for Physics for their hospitality and hiking during the production of this Letter, and we thank Matt Benacquista, Sam Finn, Vicky Kalogera, and Alberto Vecchio for organizing the LISA workshop. We are grateful to many of our fellow workshop attendees at Aspen for comments and encouragement. In particular, Kris Belczynski, Matt Benacquista, Monica Colpi, Melvyn Davies, Sam Finn, Kelly HolleyBockelmann, Vicky Kalogera, Pablo Laguna, Sterl Phinney, Tom Prince, Fred Rasio, and Dierdre Shoemaker provided many ideas in discussions, as did Steinn Sigurdsson before the workshop. We appreciate the thorough and helpful report of the anonymous referee. This Letter was supported in part by NASA grant NAG 5-13229. The work of M. F. at Northwestern University is funded through NASA ATP grant NAG 5-13236, and his participation in the Aspen Center program on LISA data was supported in part by NASA grant NNG05G106G.

\section{REFERENCES}

Bahcall, J. N., \& Wolf, R. A. 1976, ApJ, 209, 214

Barth, A. J., Greene, J. E., \& Ho, L. C. 2005, ApJ, 619, L151

Baumgardt, H., Makino, J., \& Ebisuzaki, T. 2004, ApJ, 613, 1143

Belczynski, K., Sadowski, A., \& Rasio, F. A. 2004, ApJ, 611, 1068

Bender, P. L., \& Hils, D. 1997, Classical Quantum Gravity, 14, 1439

Brown, W. R., Geller, M. J., Kenyon, S. J., \& Kurtz, M. J. 2005, ApJ, 622, L33

Farmer, A. J., \& Phinney, E. S. 2003, MNRAS, 346, 1197

Frank, J., \& Rees, M. J. 1976, MNRAS, 176, 633

Freitag, M. 2001, Classical Quantum Gravity, 18, 4033 2003, ApJ, 583, L21

Freitag, M., \& Benz, W. 2002, A\&A, 394, 345

Gould, A., \& Quillen, A. C. 2003, ApJ, 592, 935

Greene, J. E., \& Ho, L. C. 2004, ApJ, 610, 722

Gültekin, K., Miller, M. C., \& Hamilton, D. P. 2004, ApJ, 616, 221

Hamilton, D. P., \& Burns, J. A. 1991, Icarus, 92, 118 1992, Icarus, 96, 43

Hills, J. G. 1988, Nature, 331, 687 1991, AJ, 102, 704

Hils, D., \& Bender, P. L. 1995, ApJ, 445, L7

Holley-Bockelmann, K., Mihos, J. C., Sigurdsson, S., Hernquist, L., \& Norman, C. 2002, ApJ, 567, 817

Hopman, C., \& Alexander, T. 2005, ApJ, 629, 362

Hopman, C., \& Portegies Zwart, S. 2005, MNRAS, in press (astro-ph/0506181)

Hughes, S. A. 2003, Ann. Phys., 303, 142

Ivanov, P. B. 2002, MNRAS, 336, 373

Ivanova, N., Belczynski, K., Fregeau, J. M., \& Rasio, F. A. 2005, MNRAS, 358,572

Levin, Y. 2003, preprint (astro-ph/0307084)
Lightman, A. P., \& Shapiro, S. L. 1977, ApJ, 211, 244

Magorrian, J., \& Tremaine, S. 1999, MNRAS, 309, 447

Merritt, D. 2001, ApJ, 556, 245

Merritt, D., \& Ferrarese, L. 2001, ApJ, 547, 140

Merritt, D., \& Poon, M. Y. 2004, ApJ, 606, 788

Miller, M. C., \& Colbert, E. J. M. 2004, Int. J. Mod. Phys. D, 13, 1

Miralda-Escudé, J., \& Gould, A. 2000, ApJ, 545, 847

Muno, M. P., Pfahl, E., Baganoff, F. K., Brandt, W. N., Ghez, A., Lu, J., \& Morris, M. R. 2005, ApJ, 622, L113

Nelemans, G., Yungelson, L. R., \& Portegies Zwart, S. F. 2001, A\&A, 375, 890

Peters, P. C. 1964, Phys. Rev., 136, 1224

Peters, P. C., \& Mathews, J. 1963, Phys. Rev., 131, 435

Pfahl, E. 2005, ApJ, 626, 849

Poon, M. Y., \& Merritt, D. 2002, ApJ, 568, L89

Quinlan, G. D., \& Shapiro, S. L. 1989, ApJ, 343, 725

Rauch, K. P., \& Ingalls, B. 1998, MNRAS, 299, 1231

Rauch, K. P., \& Tremaine, S. 1996, NewA, 1, 149

Ryan, F. D. 1995, Phys. Rev. D, 52, 5707 1997, Phys. Rev. D, 56, 1845

Sigurdsson, S., \& Rees, M. J. 1997, MNRAS, 284, 318

Spitzer, L. 1987, Dynamical Evolution of Globular Clusters (Princeton: Princeton Univ. Press)

Syer, D., \& Ulmer, A. 1999, MNRAS, 306, 35

Tremaine, S., et al. 2002, ApJ, 574, 740

Will, C. M. 2004, ApJ, 611, 1080

Young, P. J. 1980, ApJ, 242, 1232

Yu, Q., \& Tremaine, S. 2003, ApJ, 599, 1129 